\title{
Reward signaling in a recurrent circuit of dopaminergic neurons and Kenyon cells
}

\author{
Radostina Lyutova1, Maximilian Pfeuffer ${ }^{1}$, Dennis Segebarth ${ }^{1,3}$, Jens
}

\section{Habenstein ${ }^{1,4}$, Mareike Selcho', Christian Wegener ${ }^{1}$, Andreas S.}

Thum², Dennis Pauls ${ }^{1,}$

${ }^{1}$ Neurobiology and Genetics, Theodor-Boveri Institute, Biocenter, University of

${ }^{2}$ Department of Genetics, University of Leipzig, D-04103 Leipzig, Germany.

\section{present address:}

${ }^{3}$ Institute of Clinical Neurobiology, University Hospital of Würzburg, D-97078

Würzburg, Germany

4Department of Behavioral Physiology and Sociobiology, Theodor-Boveri Institute, Biocenter, University of Würzburg, D-97074 Würzburg, Germany

${ }^{*}$ Correspondence:

Dennis Pauls, Neurobiology and Genetics, Theodor-Boveri Institute, Biocenter, 


\section{Abstract}

Dopaminergic neurons in the brain of the Drosophila larva play a key role in mediating reward information to the mushroom bodies during appetitive olfactory learning and memory. Using optogenetic activation of Kenyon cells we provide evidence that a functional recurrent signaling loop exists between Kenyon cells and dopaminergic neurons of the primary protocerebral anterior (PPAM) cluster. An optogenetic activation of Kenyon cells paired with an odor is sufficient to induce appetitive memory, while a simultaneous impairment of the dopaminergic pPAM neurons abolishes memory expression. Thus, dopaminergic PPAM neurons mediate reward information to the Kenyon cells, but in turn receive feedback from Kenyon cells. We further show that the activation of recurrent signaling routes within mushroom body circuitry increases the persistence of an odor-sugar memory. Our results suggest that sustained activity in a neuronal circuitry is a conserved mechanism in insects and vertebrates to consolidate memories.

\section{Introduction:}

Memory can be defined as a change in behavior due to experience. It enables animals and humans to adapt to a variable environment. To do so, the current situation has to be continuously re-evaluated and recorded in the brain. On the neuronal level, memories are encoded as changes in activity or connectivity which outlast the triggering environmental stimulus. Thus, the storage of relevant information in a memory trace is a multi-dimensional and dynamic process. Such complex calculations require a neuronal network with feedforward and feedback motifs enabling the system to integrate new information, to provide learned information, and to organize decision making for the behavioral outcome based on an integration of all information.

In Drosophila, the mushroom bodies (MBs) represent a multimodal integration center incorporating a variety of different sensory stimuli. A major function of the MBs is to establish, consolidate and recall associative odor memories, in both the adult and larval Drosophila ${ }^{1-8}$. During classical olfactory conditioning, odor information (CS, conditioned stimulus) is represented by an odor-specific subset of third order olfactory neurons, the MB intrinsic Kenyon cells (KCs). KCs also receive information about reward or punishment (US, unconditioned stimulus) mediated by octopaminergic 
neurons (OANs) and/or dopaminergic neurons (DANs). The coincidental activation of KCs via odor stimulation and MB input neuron (MBIN; OANs or DANs) activation leads to memory formation $1,2,4,5,9,10$.

The larval MB consist of eleven distinct compartments which are defined by the innervation pattern of MBINs and MB output neurons (MBON) ${ }^{11,12}$. The four compartments of the MB medial lobe are each innervated by a single DAN of the pPAM (primary protocerebral anterior medial) cluster. These are essential to mediate the 75 internal reward signal to the medial lobes of the MBs during odor-sugar learning ${ }^{12-14}$. In addition to the four dopaminergic pPAM neurons, OANs are also required for appetitive odor memories in the larva as they mediate the sweetness of a given sugar 15-17. Yet, it is not clear whether OANs mediate the rewarding sweetness directly to the MBs or onto the dopaminergic pPAM neurons. The finding that OANs do not innervate the medial lobes of the MBs in the larva, but potentially pPAM neurons, suggests that OANs might function directly upstream of the dopaminergic pPAM neurons as suggested in adult Drosophila ${ }^{13,16-19}$.

The current assumption is that the MBs are the brain region where synaptic plasticity occurs and from where plasticity is transferred into a conditional behavioral output driven by MBONs and downstream premotor circuits 2,4,5,10. Recently, Eichler and coworkers reconstructed the connectome of the larval MBs providing a complete neuronal and even synaptic circuit map of KCs, MBINs, and MBONs on the electronmicroscopic level ${ }^{11}$. Interestingly, this study describes a canonical circuit motif with coherent characteristics in each compartment of the MBs. This motif consists of known connections - MBINs with synaptic connections to KCs and synaptic connections of KCs onto MBONs; but it also revealed new, so far unexpected contacts: (a) reciprocal synaptic connections between KCs; (b) recurrent synaptic connections from KCs onto MBINs, and (c) direct connections from MBINs onto MBONs. So far, the function of these new connections is largely unexplored, especially in the larva.

It is, however, tempting to speculate that feedback signaling within the MB circuitry allows modulation of neuronal activity on different levels. This is supported by recent findings in adult Drosophila, where feedback signaling to MBINs is important for appetitive olfactory long-term memory formation ${ }^{20,21}$. 
Here, we experimentally address whether recently anatomically described larval KCto-DAN synapses are functional and test whether they have a role in appetitive olfactory learning in the Drosophila larva. We find that optogenetic activation of MB KCs paired with odor stimulation induces an appetitive memory, which is dependent on KC-to-DAN signaling. Moreover, calcium imaging shows that dopaminergic pPAM neurons specifically respond with an increase in $\mathrm{Ca}^{2+}$ levels to $\mathrm{MB} \mathrm{KC}$ activation. Our results indicate that a persistent recurrent activity within the neuronal network of the MBs helps to stabilize appetitive odor memories in the Drosophila larva.

\section{Results}

We used a well-established 1-odor reciprocal training regime to analyze classical olfactory learning and memory in Drosophila larvae 9,22. To address whether optogenetic activation of MB KCs affects learning and memory, we used the Gal4/UAS system ${ }^{23}$ to express Channelrhodopsin (UAS-ChR2-XXL; ${ }^{24}$ ) using the H24-Gal4

115 driver line ${ }^{25}$. H24-Gal4 specifically expresses Gal4 in all KCs and only a few neurons located in the ventral nervous system (VNS) (Fig.1A").

In contrast to the standard procedure, olfactory stimuli were paired with a blue lightdependent activation of KCs (KC-substitution learning) (Fig. 1A, $1 \mathrm{~A}^{\prime}$ ) as an artificial substitute for a conventional US like sugar or salt ${ }^{5,9}$. Strikingly, experimental larvae showed a significant appetitive memory in contrast to genetic controls that did not show any learning performance (Fig. 1 $\mathrm{A}^{\mathrm{III}}$, Fig.S1). This result suggests that the artificial activation of $\mathrm{KCs}$ is sufficient to induce an appetitive memory. To validate our findings and to exclude that the effect is based on the activation of cells of the VNS in H24>ChR2-XXL larvae, we additionally tested OK107-Gal4 (Fig. 1B) in the KCsubstitution learning experiment. Similar to H24-Gal4, OK107-Gal4 was shown to label all KCs ${ }^{26}$. Again, artificial activation of KCs using OK107-Gal4 led to an appetitive memory formation in experimental larvae (Fig. $1 \mathrm{~B}^{\mathrm{I}}$ ). Thus, KC-substitution learning using two MB-specific Gal4 lines confirmed that optogenetic activation of $\mathrm{KCs}$ is sufficient to induce an appetitive memory (Fig. 1A, 1B). 

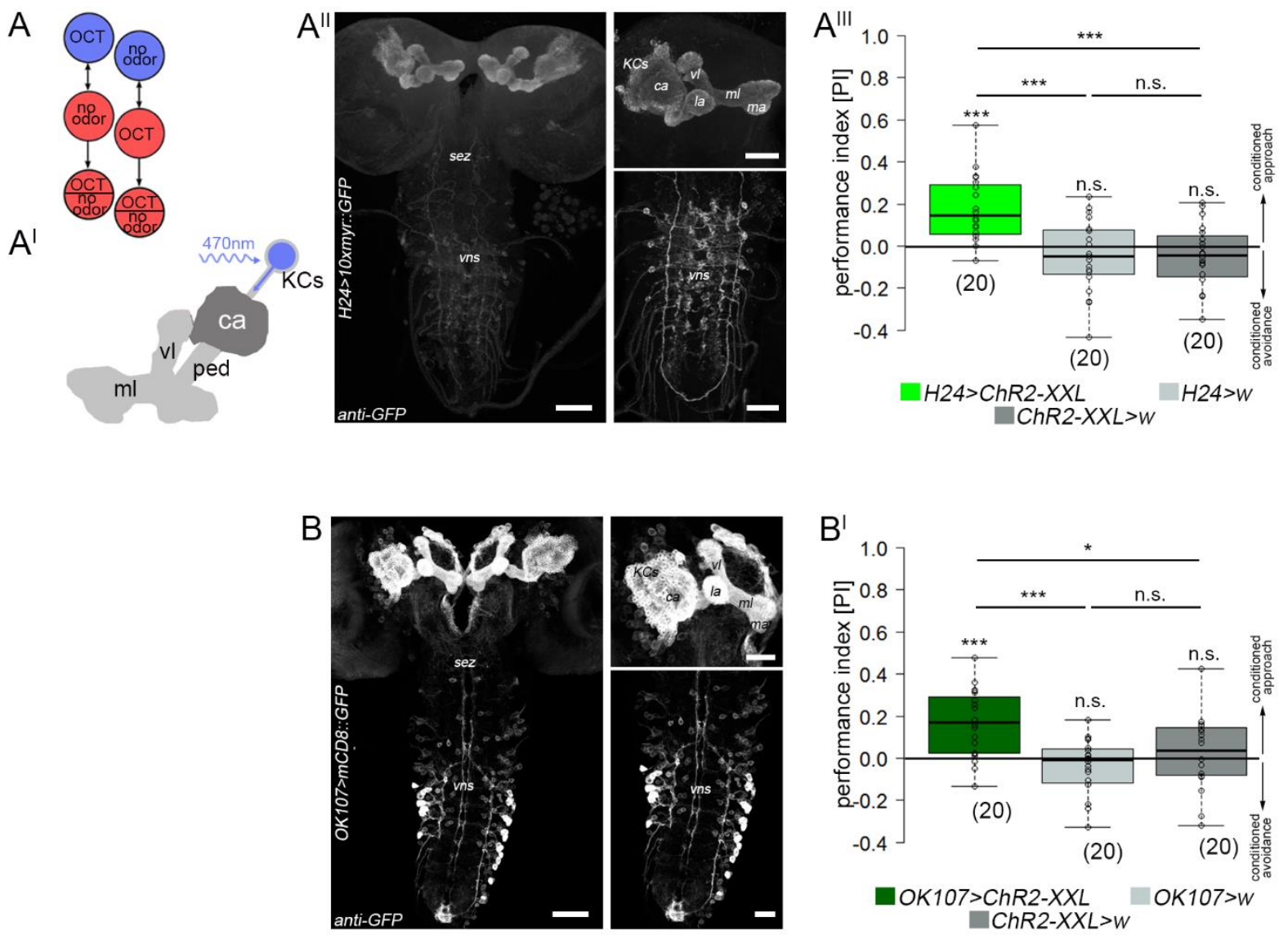

Fig. 1: Optogenetic activation of mushroom body Kenyon cells during conditioning is sufficient to induce appetitive memory formation. (A) Illustration of the 1-odor reciprocal training regime used in this study (substitution learning). ( $\left.A^{\prime}\right)$ Schematic drawing of the larval $M B$ expressing ChR2-XXL in all KCs. ( $\left.A^{\prime \prime}\right)$ Expression pattern of H24-Gal4 crossed with 10xUAS-myr::GFP stained with anti-GFP (white). H24-Gal4 shows expression in the complete

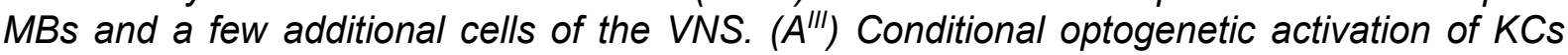
coupled to odor information during training is sufficient to induce an appetitive memory. (B) Expression pattern of OK107-Gal4 crossed with UAS-mCD8::GFP stained with anti-GFP (white). ( $\left.B^{\prime}\right)$ Conditional optogenetic activation of KCs coupled to odor stimulation during training is sufficient to induce an appetitive memory expression in OK107-Gal4. ca: calyx; ChR2-XXL: channelrhodopsin2-XXL; GFP: green-fluorescent protein; KCs: Kenyon cells; la: lateral appendix; ma: medial appendix; ml: medial lobe; OCT: octanol; ped: peduncle; sez: subesophageal zone; vl: vertical lobe; vns: ventral nervous system; $w$ : $w^{1118}$. Significance levels: $p>0.05$ n.s., $p<0.05^{*}, p<0.01^{* *}, p<0.001^{* * *}$. Scale bars: $50 \mu m$ and $25 \mu m$ for higher magnifications.

\section{Optogenetic activation of mushroom body Kenyon cells does not affect larval locomotion or innate odor responses}

150 The MBs integrate a variety of different sensory modalities. To exclude that the optogenetic activation of KCs alters general behaviors like locomotion or the processing of sensory stimuli necessary for classical olfactory learning (i.e. odors), larvae were assayed for (i) locomotion and (ii) innate odor preference. We analyzed larval locomotion using the FIM tracking system ${ }^{27}$. For this, larvae were monitored under red light for one minute. Subsequently, larvae were exposed to blue light and 
recorded again for one minute (Fig. 2; the light regime is indicated by the red and blue rectangles). Optogenetic activation of KCs did not affect the velocity and crawled distance over time of $H 24>C h R 2-X X L$ larvae, suggesting that artificial activation of KCs does not affect general locomotion parameters (Fig. 2A-A", Fig. S2). However, experimental larvae showed a significant decrease in the number of turns and stops under blue light exposure, suggesting a change in orientation behavior (Fig. S2', S2"l). Next, we tested whether the optogenetic activation of KCs alters innate odor preference, which would perturb the conditioning experiments of our study. For this, larvae were assayed in a simple choice test using pure octanol (OCT; the odor used in our KC-substitution learning) and diluted amylacetate (AM; commonly used for the twoodor reciprocal experimental design; ${ }^{5,9}$ ), respectively. Optogenetic activation of KCs in experimental larvae did not change the innate preference to OCT and AM (Fig. 2B, $\left.2 \mathrm{~B}^{\prime}\right)$. To verify that the activation of all $\mathrm{KCs}$ does not change odor quality to an unspecific attractive odor, larvae were tested in a choice test with exposure to OCT and AM opposing each other. Previous studies showed that pure OCT and 1:40 diluted AM are balanced in their attractiveness to larvae, while larvae prefer pure AM over pure OCT. As expected, experimental larvae showed a significant approach towards $\mathrm{AM}$, which was indistinguishable from controls (Fig. 2B" ); coherently both experimental and control larvae were randomly distributed in the choice test between OCT and 1:40 diluted AM (Fig. 2B ${ }^{\text {III }}$ ). These results suggest that an optogenetic activation of all KCs does not change innate odor preferences. We also repeated the KC-substitution learning experiment using the two-odor reciprocal design (Fig. 2C; ${ }^{5,9}$ ). Similar to our initial results, optogenetic activation of all KCs was sufficient to induce appetitive memory, verifying that odor discrimination is not disturbed due to artificial activation of KCs via ChR2-XXL (Fig. $2 \mathrm{C}^{\prime}$ ). Taken together, these results suggest that optogenetic activation of KCs does not perturb locomotion and innate preference to odors essential for KC-substitution learning. 

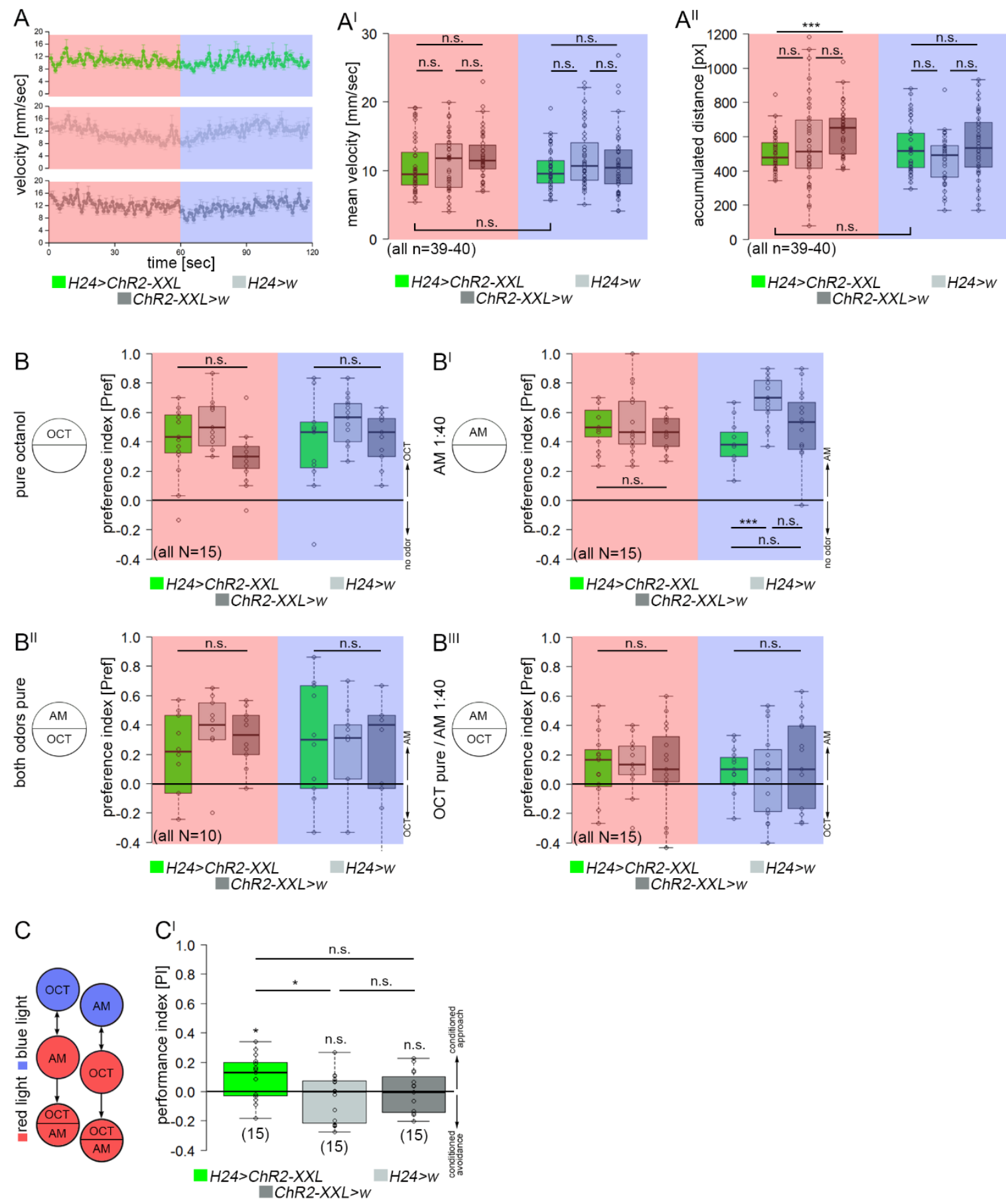

185 Fig. 2: Optogenetic activation of mushroom body Kenyon cells does not alter locomotor behavior or innate odor preference in larvae. (A) Conditional optogenetic activation of KCs does not change larval velocity $\left(A\right.$ and $\left.A^{\prime}\right)$ and accumulated distance $\left(A^{\prime \prime}\right)$. The light regime is indicated by the red and blue rectangles: larvae were monitored under red light for one minute, subsequently under blue light for another minute. (B) Similarly, innate odor preference to OCT

190 (B) and diluted $A M\left(B^{\prime}\right)$ is not altered due to conditional optogenetic activation of KCs. In line, experimental larvae showed normal performance in an odor discrimination task using OCT and either pure $A M\left(B^{\prime \prime}\right)$ or 1:40 diluted $A M\left(B^{\prime \prime \prime}\right)$. (C and $\left.C^{\prime}\right)$ Conditional optogenetic activation of $K C s$ is sufficient to induce an appetitive memory using a 2-odor reciprocal training regime indicating that experimental larvae can distinguish different odors despite artificially activated KCs. AM: amylacetate; ChR2-XXL: channelrhodopsin2-XXL; KCs: Kenyon cells; OCT: octanol; $w: w^{1118}$. Significance levels: $p>0.05$ n.s., $p<0.05^{*}, p<0.01^{* *}, p<0.001^{* * *}$. 


\section{Why is the activation of KCs sufficient to specifically induce appetitive memory?} Our results so far illustrate that artificial activation of KCs paired with odor exposure is sufficient to specifically induce appetitive memory. Therefore, we tested whether the reward signal would be sufficient to induce an associative reward memory in our KCsubstitution learning experiment if tested on a test plate without US information (Fig. 1A). We tested larvae in a simple light preference test. Feeding larvae strongly avoid light to ensure staying within the food ${ }^{28-30}$. Thus, we challenged $H 24>C h R 2-X X L$

205 larvae to choose between blue light and darkness. Given the idea that the activation of KCs induces internal reward signaling, we assumed that control larvae would prefer darkness over blue light, whereas $H 24>C h R 2-X X L$ larvae would show a reduced dark preference as they would experience blue light as reward (Fig. 3A). As expected, control larvae significantly avoided the illuminated rectangles after $1 \mathrm{~min}$ and $3 \mathrm{~min}$. In

210 contrast, experimental larvae showed light avoidance only after $3 \mathrm{~min}$, but were randomly distributed after $1 \mathrm{~min}$ suggesting that the experimental larvae showed a delayed light avoidance (Fig. $3 A^{\prime}$ ). To verify that larvae are challenged to decide between reward and innate light avoidance in our assay, we repeated the experiment, but this time used red light, which does not elicit innate light avoidance in the larva 31,32

215 (Fig. 3A"), but activates KCs in experimental larvae as we expressed UAS-Chrimson, a red-light driven channelrhodopsin ${ }^{33}$. H24>Chrimson larvae showed a significant preference for the red-light illuminated rectangles within the first minutes, while control larvae were randomly distributed. Similar to our previous results, the effect was gone after $3 \mathrm{~min}$ (Fig. $3 \mathrm{~A}^{\mathrm{III}}$ ). To test this even further, we challenged the larvae to decide

220 between OCT in the darkness and blue light at the opposing side (Fig. S4, S4'). In this assay, control larvae are driven to go into the darkness due to their innate light avoidance and innate OCT preference. Control larvae strongly preferred the dark and OCT side over blue light. Experimental larvae, however, showed significantly reduced preference for darkness and OCT. This supports our hypothesis that experimental 225 larvae were challenged to decide between their innate OCT and darkness preference versus an artificially induced internal reward signaling in the blue light (Fig. S4'). Taken together, our results suggest that optogenetic activation of KCs elicits an internal reward signaling which induces appetitive memory during KC-substitution learning. This is further supported by the change in orientation behavior, reflected by the 230 reduction in turning rate and direction (Fig. S2', S2"l), in larvae with optogenetically 
activated KCs. Blue light exposure without any gradient may provide an even distributed pleasant environment in experimental larvae due to the optogenetic activation of internal reward signaling. Consequently, experimental larvae reduce turning rate and turning direction, but not the speed (Fig. S2', S2" ) ${ }^{34}$.

235 In our experiments larvae are tested on pure agarose, thus they will just expect a significant gain through the expression of appetitive memory. We argue that the lack of aversive olfactory memory expression is based on the test situation in our KCsubstitution learning experiment, as we test larvae intentionally on pure agarose (Fig. 1A). Drosophila larvae are known to express aversive memories only in the presence

240 of the aversive US in the test situation ${ }^{5,9,35}$. In contrast, to recall appetitive memories the appetitive US must be absent during the test (Fig. S3). Larvae compare the value of a given US in the test situation with the one used during training and only an expected gain will drive the conditional output behavior $5,9,36$.

\section{Dopaminergic neurons of the pPAM cluster mediate internal reward signals}

Previous studies have shown that dopaminergic cells of the pPAM cluster in the larval and adult Drosophila mediate internal reward signals during associative conditioning $12,13,16,37$. To test whether the internal reward signaling described in our study is based on the activity of pPAM neurons, we repeated the light avoidance experiment, but this

250 time expressed ChR2-XXL via R58E02-Gal4 which labels 3 out of 4 neurons of the larval pPAM cluster (Fig. 3B" ${ }^{13}$. In line with our previous results, optogenetic activation of pPAM neurons abolished light avoidance in R58E02>ChR2-XXL larvae (Fig. $3 \mathrm{~B}^{\prime}$ ). The effect was even stronger compared to $H 24>C h R 2-X X L$ larvae (Fig. $3 A^{\prime}$ ), as R58E02>ChR2-XXL larvae showed no light avoidance after both $1 \mathrm{~min}$ and $3 \mathrm{~min}$ (Fig. $\left.3 \mathrm{~B}^{\prime}\right)$. Further, we tested whether the pPAM-dependent reward signaling is induced by the optogenetic activation of KCs in $H 24>C h R 2-X X L$ larvae. We used the lexA/LexAop system ${ }^{38}$ together with the Gal4/UAS system to activate KCs (H24>ChR2-XXL) and simultaneously ablate pPAM neurons (R58E02>reaper). As expected, optogenetic activation of KCs abolished the innate light avoidance in both genetic controls

260 (H24>ChR2-XXL,R58E02>w and H24>ChR2-XXL;reaper>w) after $1 \mathrm{~min}$ and $3 \mathrm{~min}$ (Fig. $3 C^{\prime}$ ). In contrast, experimental larvae with ablated pPAM neurons showed light avoidance after $3 \mathrm{~min}$, indicating that optogenetic activation of $\mathrm{KCs}$ induces internal reward signaling via the activation of pPAM neurons. These results are supported by the electron-microscopic reconstruction of the larval MBs, which described a canonical 
265 circuit motif in each compartment including direct KC-to-MBIN synaptic connections ${ }^{11}$. In line, we used the GRASP technique which allows to reconstitute potential synaptic connections as GFP fluorescence is only visible when the neurons of interest are in close vicinity ${ }^{39}$. GFP fluorescence was exclusively detectable in the MBs, mostly in the medial lobe which is the main output region of the pPAM neurons (Fig. $\left.3 C^{\prime \prime}\right)^{13}$. This

270 supports the hypothesis that the optogenetic activation of KCs induces pPAMdependent reward signaling directly at the level of the MBs, as no signal was elsewhere detectable.
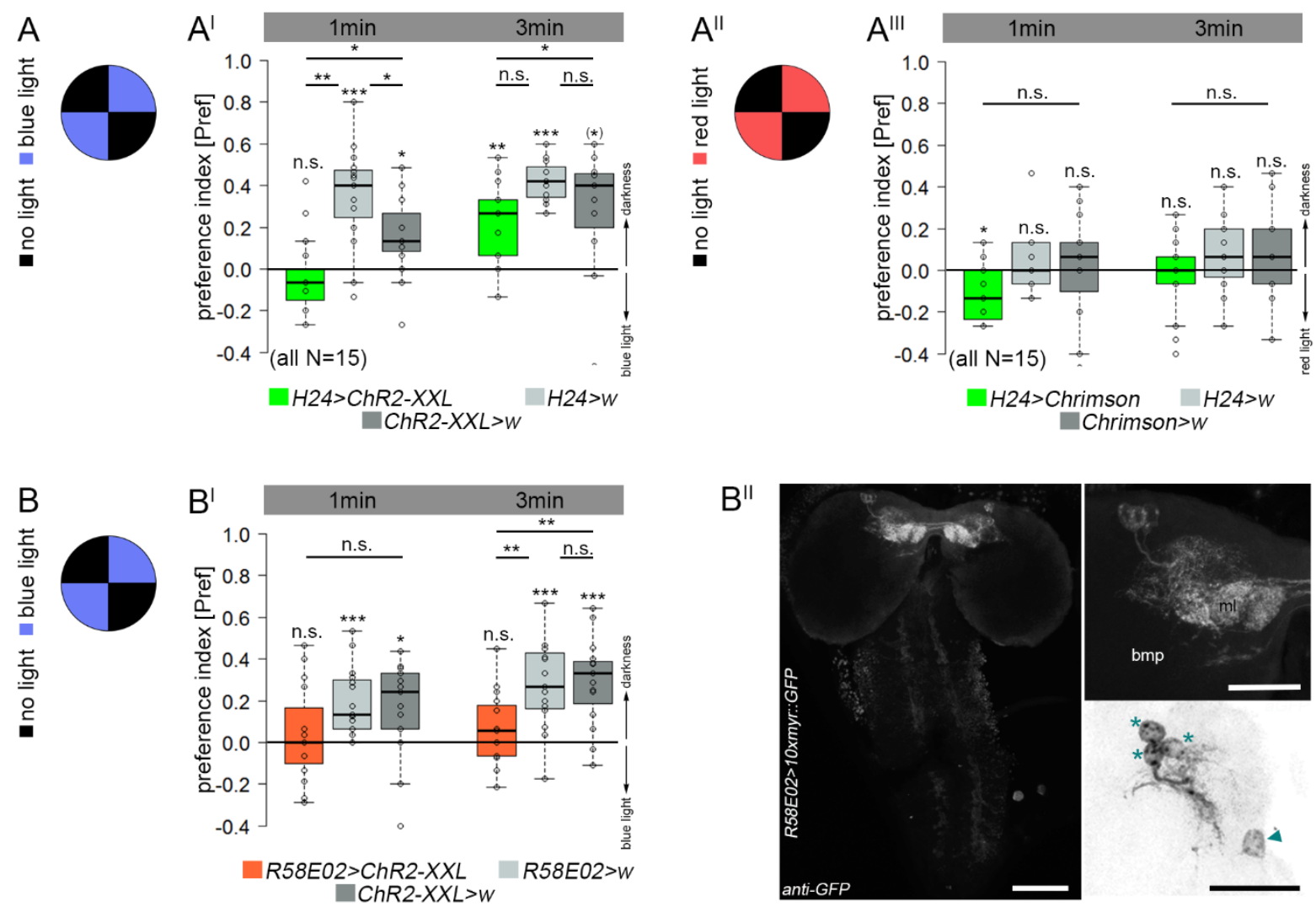

$\mathrm{B}^{\prime \prime}$
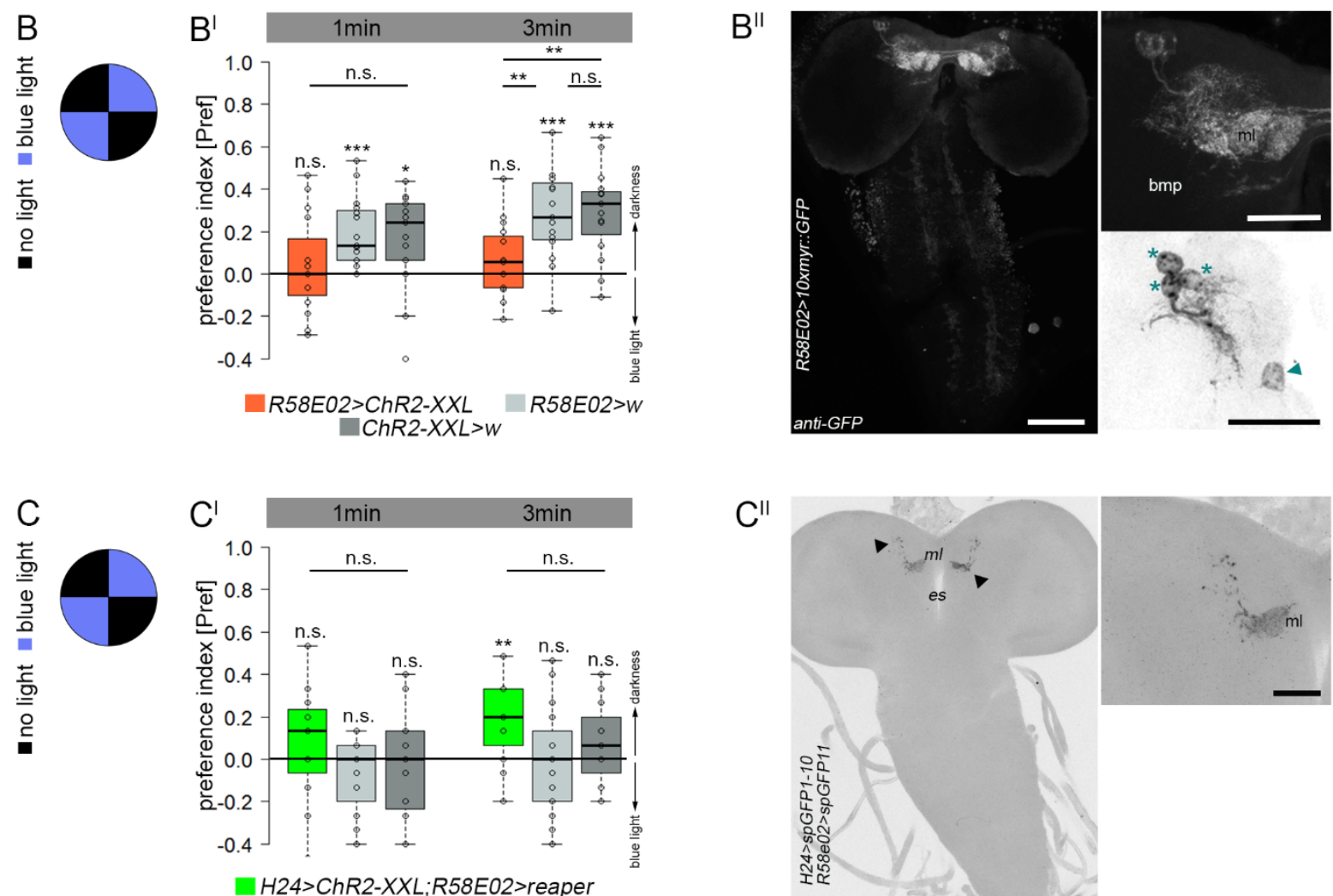

$\mathrm{C}^{\prime \prime}$

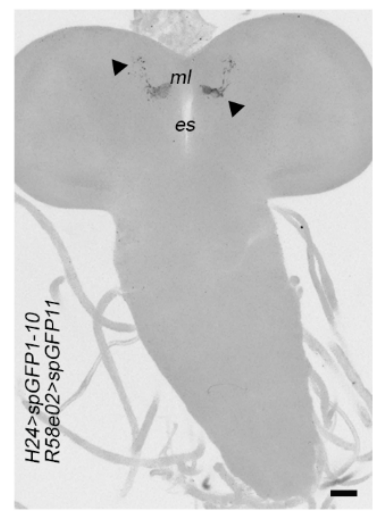

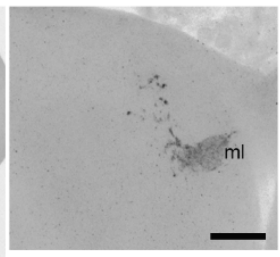


Fig. 3: Optogenetic activation of mushroom body Kenyon cells induces internal reward signaling via dopaminergic neurons of the pPAM cluster. Control larvae showed blue light avoidance in a simple choice test (A). H24>ChR2-XXL larvae do not show any light avoidance after $1 \mathrm{~min}$, but after $3 \mathrm{~min}\left(A^{\prime}\right)$ suggesting that the blue light-dependent activation of ChR2-XXL induces internal reward signaling. ( $A^{\prime \prime}$ and $\left.A^{\prime \prime \prime}\right)$ In line, $H 24>C$ Chrimson larvae prefer red light over darkness after $1 \mathrm{~min}$, while genetic controls were distributed randomly. $\left(B+B^{\prime}\right)$ Optogenetic activation of pPAM neurons using R58E02-Gal4 abolished light avoidance. ( $\left.B^{\prime \prime}\right)$ Expression pattern of R58E02-Gal4 crossed with 10xUAS-myr::GFP and stained with anti-GFP (white). R58E02-Gal4 shows specific expression in three DANs of the pPAM (protocerebral anterior

290 medial) cluster (asterisks) and one additional cell body at the midline (arrow head). pPAM neurons innervate the medial lobe of the MBs. ( $C$ and $C^{\prime}$ ) Optogenetic activation of KCs abolished light avoidance in both genetic controls; in contrast experimental larvae with ablated pPAM neurons showed light avoidance after $3 \mathrm{~min}$, indicating that optogenetic activation of KCs induces internal reward signaling via pPAM neurons. $\left(C^{\prime \prime}\right)$ Reconstituted split-GFP (with anti-

295 GFP) between H24-Gal4 positive neurons and R58E02-Gal4 positive neurons is only visible at the level of the mushroom bodies (arrowheads). bmp: basomedial protocerebrum; ChAT: cholinacetyltransferase; ChR2-XXL: channelrhodopsin2-XXL; DANs: dopaminergic neurons; Fasll: fasciclinll; KCs: Kenyon cells; MBs: mushroom bodies; ml: medial lobe; es: esophagus; $w$ : $w^{1118}$. Significance levels: $p>0.05$ n.s., $p<0.05^{*}, p<0.01^{* *}, p<0.001^{* * *}$. Scale bars: $50 \mu m$ 300 and $25 \mu \mathrm{m}$ for higher magnifications.

\section{Kenyon cell to dopaminergic neuron signaling is sufficient to establish an appetitive memory}

Our results suggest that the optogenetic activation of KCs induces reward signaling 305 involving a recurrent pPAM cluster loop. Previous studies already showed that the DANs of the pPAM cluster are important MBINs to establish larval olfactory memories in the Drosophila larva ${ }^{12,13}$. Thus, we tested whether the recurrent KC-to-pPAM loop is important to establish the appetitive memory observed in our KC-substitution learning experiment (Fig. 1 $\mathrm{A}^{\mathrm{III}}$ ). First, we tested whether the ablation of pPAM neurons 310 affects appetitive memory established via optogenetic activation of KCs (H24>ChR2$X X L ; R 58 E 02>$ reaper). Indeed, experimental larvae showed no appetitive memory in contrast to the genetic controls (Fig. 4A'). Second, to verify these results, we activated the KCs and simultaneously downregulated the dopamine receptor DopR1 (dcr,H24>ChR2-XXL;DopR1-RNAi) in all KCs. DopR1 is necessary for appetitive 315 olfactory learning ${ }^{14,40}$. Experimental larvae showed no appetitive memory in contrast

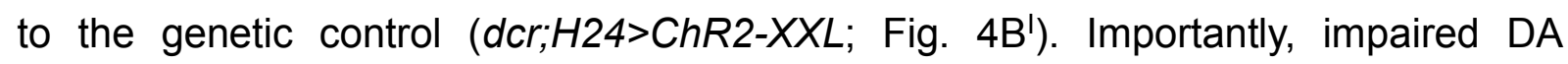
signaling did not affect learning-relevant odor preferences (Fig. 4A", 4B"). Next, we monitored changes of intracellular $\mathrm{Ca}^{2+}$ levels in R58E02-positive pPAM neurons upon optogenetic activation of KCs (H24>ChR2-XXL;R58E02>GCamp6m). Isolated brains 
were first exposed with a low blue light intensity $\left(\sim 700 \mu \mathrm{W} / \mathrm{cm}^{2}\right)$ to monitor baseline activity; subsequently, brains were exposed with a higher light intensity $\left(\sim 3800 \mu \mathrm{W} / \mathrm{cm}^{2}\right)$. This light intensity was sufficient to induce a significant increase in $\mathrm{Ca}^{2+}$ levels within pPAM neurons due to the activation of ChR2-XXL in KCs, which confirms the functionality of the KC-to-PAM connection (Fig. 4C: exemplary responses; $\left.4 \mathrm{C}^{\prime}\right)$. Coherently, we found a similar increase in intracellular $\mathrm{Ca}^{2+}$ levels in pPAM neurons due to the optogenetic activation of KCs by using UAS-Chrimson (Fig. S5, $\left.\mathrm{S}^{\prime}\right)$. Taken together, our results suggest that functional recurrent signaling between MB KCs and pPAM neurons exist in the Drosophila larva.
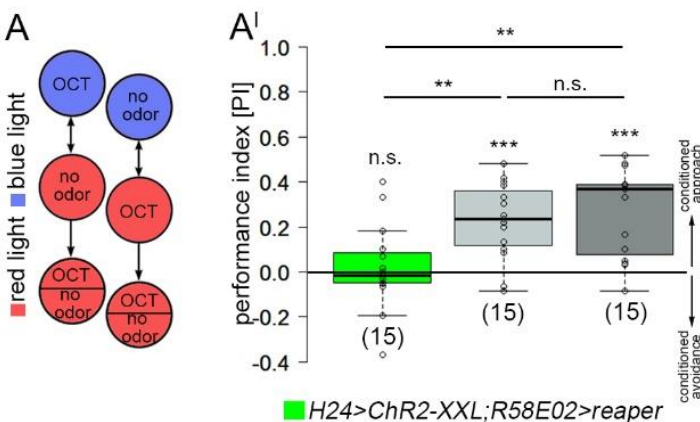

H24>ChR2-XXL;R58E02>W H24>ChR2-XXL;reaper>W

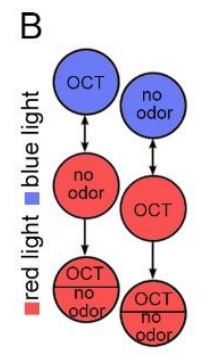

$\mathrm{B}^{\mathrm{I}}$

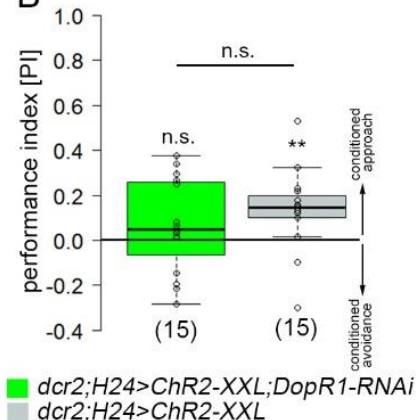

dcr2;H24>ChR2-XXL;DopR1-RNA dcr $2 ; H 24>C h R 2-X X L$
A

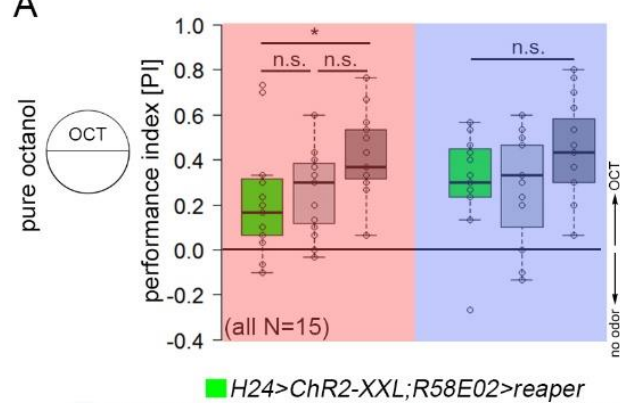

$H 24>C h R 2-X X L ; R 58 E 02>W \quad H 24>C h R 2-X X L ;$ reaper $>W$

$\mathrm{B}^{\prime \prime}$

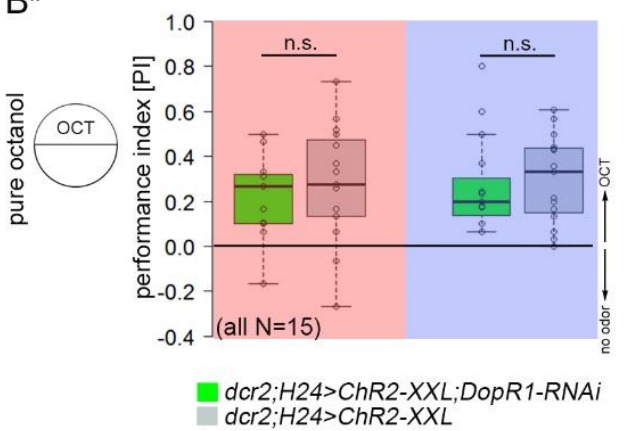

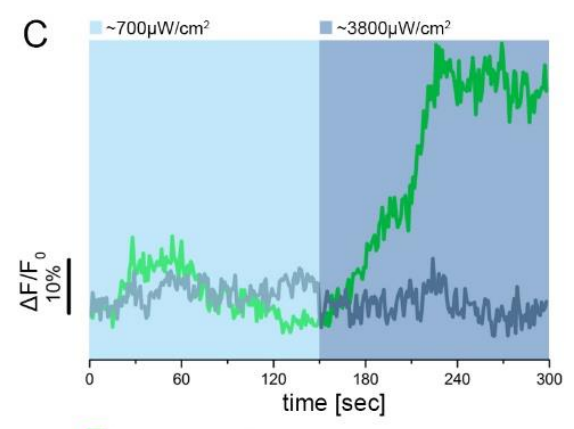

H24>ChR2-XXL;R58E02>GCamp6m ChR2-XXL>W;R58E02>GCamp6m

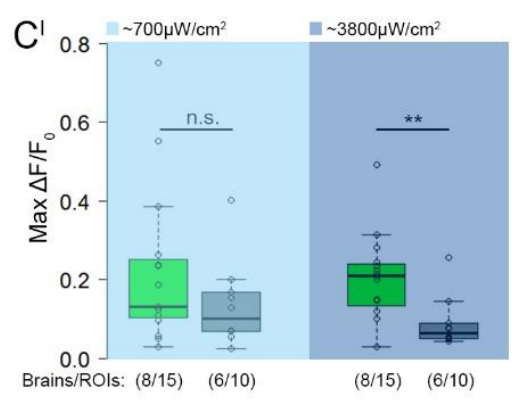

H24>ChR2-XXL;R58E02>GCamp6m ChR2-XXL>W;R58E02>GCamp6m 
Fig. 4: Appetitive memory expression due to the optogenetic activation is based on Kenyon cells to dopaminergic pPAM neurons signaling

$\left(A\right.$ and $\left.A^{\prime}\right)$ Optogenetic activation of KCs and simultaneous ablation of pPAM neurons via the expression of the apoptotic gene reaper impairs optogenetically induced appetitive memory.

$\left(A^{\prime \prime}\right)$ Innate odor preference is unchanged in experimental larvae under red and blue light. ( $B$ and $\left.B^{\prime}\right)$ Similarly, optogenetic activation of KCs and simultaneous knockdown of the dopamine receptor DopR1 impairs appetitive memory formation. $\left(B^{\prime \prime}\right)$ Innate odor preference is unchanged in experimental larvae under red light (red rectangle) and blue light (blue rectangle). ( $C$ and $\left.C^{\prime}\right)$ pPAM neurons respond with a significant increase in intracellular $\mathrm{Ca}^{2+}$

350 levels $\left(C^{\prime}\right)$ to an optogenetic activation of MB KCs (green; illumination with blue light (475nm)) verifying a functional KC-to-DAN feedback connection. An increase in $\mathrm{Ca}^{2+}$ levels was absent in genetic controls (grey) indicating that the response in the experimental brains was specific to the optogenetic activation rather than to light per se. ChR2-XXL: channelrhodopsin2-XXL; KCs: Kenyon cells; MBs: mushroom bodies; OCT: octanol; $w$ : $w^{1118}$. Significance levels: $p>0.05$ n.s., $p<0.05^{*}, p<0.01^{* *}, p<0.001^{* * *}$.

\section{Reward signaling in the recurrent MB circuit stabilizes appetitive memories over time}

360 The larval MB connectome revealed a complex canonical circuit motif in each MB compartment, with suggested MBIN-to-KC and KC-to-MBON synapses, but also unknown recurrent KC-to-MBIN and MBIN-to-MBON synapses ${ }^{11}$. Our data suggests that the KC-to-MBIN connection is functional, which is in line with a recent report in adult Drosophila ${ }^{41}$. To test whether KC-to-pPAM feedback signaling affects normal

365 appetitive olfactory learning, we exposed experimental larvae to a real sugar stimulus and a simultaneous optogenetic activation of KCs via ChR2-XXL, while control flies were trained with sugar only under red light without any blue light exposure (Fig. 5A). $H 24>C h R 2-X X L$ larvae trained to associate OCT with sugar under red light (normal sugar learning) showed significant appetitive memory expression immediately after 370 training. However, memory expression was abolished $15 \mathrm{~min}$ after training (Fig. 5A", 5B). $H 24>C h R 2-X X L$ larvae that received sugar stimulation in combination with artificial KC activation during training showed comparable appetitive memory expression immediately after training. However, the appetitive memory was still detectable 45min after training, suggesting a change in memory persistence based on 375 artificial activation of the recurrent $M B$ network (Fig. 5A", 5B). Optogenetic activation of KCs did not change innate preference for sugar in $H 24>C h R 2-X X L$ larvae (Fig. S6, $\left.S 6^{\prime}\right)$. 
A

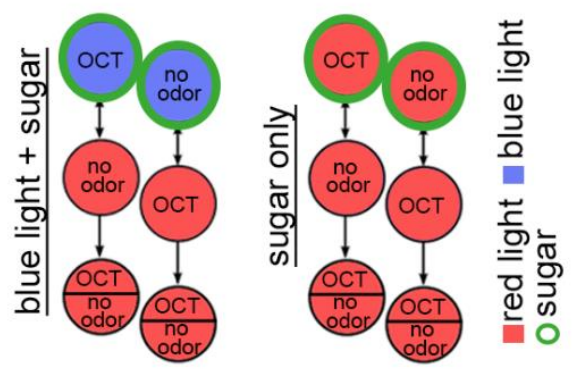

$A^{\prime}$
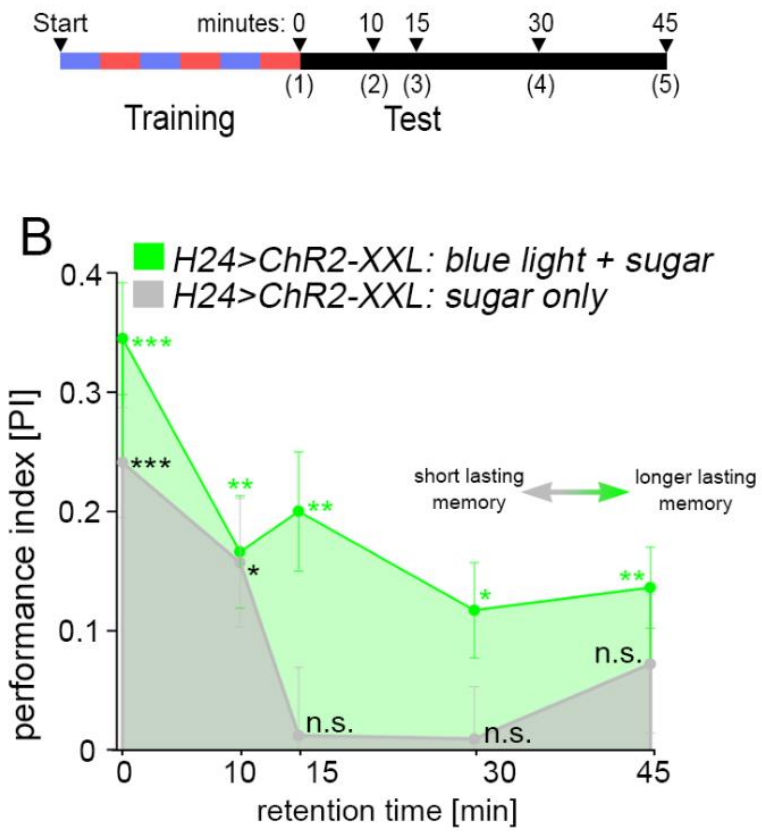

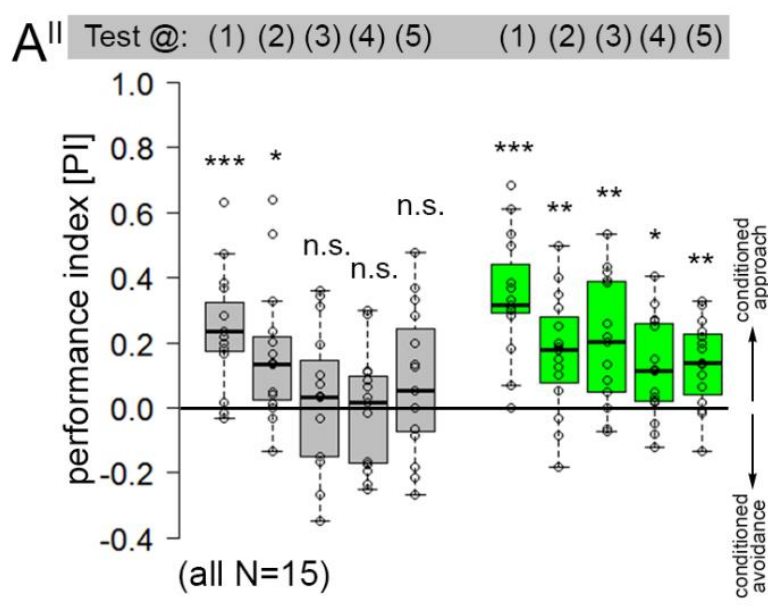

H24>ChR2-XXL: blue light + sugar H24>ChR2-XXL: sugar only

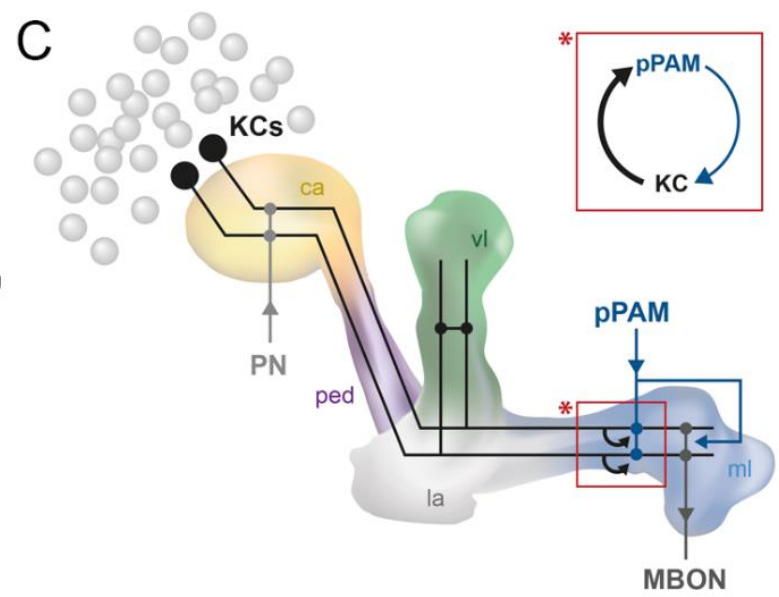

Fig. 5: Sustained activity in the mushroom body circuit increases memory stabilization. $\left(A\right.$ and $\left.A^{\prime}\right)$ Schematic drawing of the training and test regime: H24>ChR2-XXL larvae were either trained for odor-sugar associations under red light, or for odor-sugar associations with paired artificial KC activation (green circle). Appetitive memory was either tested immediately after training, or 10min, 15min, 30min and 45min after training. ( $\left.A^{\prime \prime}, B\right) H 24>C h R 2-X X L$ larvae trained under red light ("sugar only"; grey) showed significant performance scores immediately after training as well as $10 \mathrm{~min}$ after training. No memory was detectable $15 \mathrm{~min}$ or later after training. $\mathrm{H24}>\mathrm{ChR2-XXL}$ larvae trained with blue light exposure and sugar stimulus during training ("blue light + sugar"; green) showed significant memory 45min after training. (C) Schematic of the mushroom body circuit focusing on the connectivity with dopaminergic input neurons. Recurrent signaling between Kenyon cells and dopaminergic PPAM neurons exists enabling ongoing activity of the dopaminergic neurons and facilitation of KC-to-MBON (KC: Kenyon cell, MBON: mushroom body output neuron) signaling. ChR2-XXL: channelrhodopsin2-XXL; ca: calyx; KCs: Kenyon cells; la: lateral appendix; MBON: mushroom body output neuron; ml: medial lobe; OCT: octanol; ped: peduncle; pn: projection neuron; vl: vertical lobe. Significance levels: $p>0.05$ n.s., $p<0.05^{*}, p<0.01^{* *}, p<0.001^{* * *}$. 


\section{Discussion}

The MBs are multimodal integration centers. Beside their main function in olfactory 405 learning and memory, MBs are involved in the control of locomotor behavior, sleep, visual learning, courtship conditioning, as well as context-dependent associations ${ }^{42}$. Also in the Drosophila larva, several studies accurately demonstrated a role of MB KCs for olfactory learning and memory ${ }^{5}$ : classical genes involved in learning and memory like rutabaga and dunce, both part of the cAMP signaling pathway, are expressed in

410 larval KCs ${ }^{43,44}$. Mutants for both genes are impaired in olfactory learning and memory. Moreover, input into the MBs is required to establish a memory during training, while output of MB KCs is necessary for memory retrieval ${ }^{3,44}$. These and other findings allowed to postulate a minimal and functional MB network model. However, the recent larval MB connectome described several novel signaling routes besides the classical

415 MBIN-to-KC-to-MBON circuit ${ }^{11}$, which is in line with new data for the adult MB circuit 20,41,45-47. Especially the role of MB KCs has been underestimated, as they form KCto-KC synapses and provide a recurrent loop with MBINs. As the function of this recurrent loop is not yet known in larvae, we explored the function of this new circuit motif for its role in larval olfactory learning and memory in this study.

Using two different MB-specific driver lines we found that optogenetic KC activation is sufficient to induce olfactory appetitive memory. The artificial activation of all KCs did not alter odor processing, sugar processing, or larval locomotion. Based on these findings, we suggest to extend the current appetitive olfactory learning circuitry in the 425 following way: during larval odor-sugar conditioning, CS (odor) information is mediated by projection neurons to the MB calyx, while simultaneously US (sugar) information is mediated via dopaminergic pPAM neurons to the medial lobe. The coincident odordependent $\mathrm{Ca}^{2+}$ influx and DA release induces cellular plasticity (in terms of shortlasting memories; this study) or synaptic plasticity between KCs and MBONs (for 430 longer-lasting memories) ${ }^{4}$. Our new data suggest, that in addition pPAM neurons receive input from $\mathrm{KCs}$ during training. Optogenetic activation of KCs was sufficient to activate the dopaminergic PPAM neurons indicating a functional connection of KCs-topPAM, which was anatomically identified in the larval MB connectome ${ }^{11}$. However, whether the KC-to-pPAM signaling is direct or indirect remains still elusive. Yet, during odor-sugar training a recurrent circuit of $\mathrm{KCs}$ and pPAMs could become active, instruct 
and consolidate an appetitive olfactory memory. Indeed, when this loop was blocked, either through DopR1 knockdown in KCs or ablation of pPAM neurons, the induction of appetitive memory by optogenetic activation of KCs was impaired.

440 In adult Drosophila, activity in dorsal paired median neurons is necessary during the consolidation phase after training to form a persistent memory ${ }^{48}$. However, also during training ongoing activity and feedback signaling is necessary for olfactory learning and memory as impaired cholinergic input from KCs to dopaminergic MBINs reduces odorelectric shock memories in adult Drosophila ${ }^{41}$. In this sense, KC-to-DAN feedback

445 signaling seems to affect neuronal activity in MBINs and by that may enhance the release of DA to the MBs beyond the level induced by the US stimulus alone ${ }^{41}$. Likewise, dopaminergic MBINs receive excitatory input from MBONs during courtship training and training for odor-sugar associations, which in turn leads to a persistent activity of these DANs and an increase in DA release onto the MBs. Thus, MBONs are

450 not only necessary for memory retrieval, but also for memory acquisition and consolidation. Consequently, a longer-lasting memory is formed by the facilitation of KC-to-MBON signaling through the recurrent MBON-to-DAN feedback loop ${ }^{20,47}$. Taken together, within the MB circuitry direct recurrent KC-to-DAN loops together with one step recurrent KC-MBON-DAN loops exist to shift an initial nascent memory into a 455 stable memory.

\section{Do recurrent circuits within the larval mushroom bodies allow the formation of different types of memories?}

Our results indicate that a paired exposure to sugar and optogenetic activation of KCs

increases the persistence of odor-sugar memory. Larvae trained for only odor-sugar associations under red light showed memory expression up to $10 \mathrm{~min}$. $15 \mathrm{~min}$ after training the appetitive olfactory memory was abolished. On the contrary, artificial activation of KCs combined with sugar exposure during training was sufficient to induce a longer-lasting appetitive olfactory memory. Memory expression was still detectable 465 45min after training. Interestingly, in adult Drosophila activity in dopaminergic MP1 neurons is necessary in the first $30-45 \mathrm{~min}$ after training to trigger long-term memory formation 21,49 . Thus, we assume that an increased and persistent activity within the MB network, including the KC-to-pPAM recurrent circuit signaling, may likewise support the formation of longer-lasting memories in the Drosophila larva. It is tempting 
470 to speculate that the activity level in dopaminergic pPAM neurons, which is increased via the KC-to-pPAM signaling through optogenetic activation of KCs in our study, is crucial to drive the conditional behavioral output. Different activity levels set a specific level of intracellular cAMP, either triggering a short-lasting memory (by a cAMPdependent transient increase in protein kinase $A(P K A)$ ) or longer-lasting memories (by

475 more stable cAMP-dependent elevation of PKA) ${ }^{50,51}$. Changes in DA neuronal activity (presumably controlled by the existing feedback loops) may provide a steady update about the internal state and the expected gain from memory expression based on available environmental stimuli. The type of internal information may thus be appropriate to regulate the type of memory which is formed. In short, under food restriction adult flies preferentially show anesthesia-resistant memory rather than longterm memory, as this consolidated memory form is independent of energy-demanding protein biosynthesis and therefore at low cost ${ }^{52,53}$. Further, in both flies and larvae, neurons expressing the neuropeptide dNPF (Drosophila neuropeptide F; an orthologue of mammalian NPY) modulate memory processes to match information about the 485 current reward stimulus with the internal state of the animal. A conditional activation of dNPF neurons during training reduced the acquisition of odor-sugar memories in the Drosophila larva ${ }^{54}$. Odor-sugar learning in flies is most efficient when flies are food deprived. dNPF neurons modulate the expression of odor-sugar memories via feedforward inhibition through dopaminergic MBINs and the MBON circuitry. In hungry 490 flies, specific MBONs (MV2) are inhibited by the action of dNPF neurons through MP1 DANs, which elicits the expression of odor-sugar memories and thus conditional approach behavior ${ }^{55}$.

Thus, DANs innervating different compartments of the MBs may provide a dynamic intrinsic and extrinsic neuromodulatory system: depending on experience and the 495 internal state, the same sensory input (e.g. a sugar stimulus) may drive a different conditional behavioral output (either approach, avoidance or indifference) and different temporal forms of associative olfactory memories (short-term memory, anesthesiaresistant memory or long-term memory) ${ }^{45,56}$.

Our assumption is now that a recurrent signaling loop within the larval MB circuit allows 500 the larva to constantly adapt appetitive memory formation and retrieval to the current internal state and external situation, mainly based on the modulation of ongoing activity in pPAMs, and by that to incorporate whether the expected gain is sufficient to store and recall a long-lasting associative memory. In humans, DA was shown to modulate 
a post-encoding consolidation process with respect to episodic memory persistence

505 57. The molecular consolidation process is based on DA-dependent protein-synthesis, which elicits long-term plasticity in the hippocampus. Similarly, in rodents the activation of different DA receptors in the hippocampus is critical at or around the time of memory encoding to modulate memory persistence ${ }^{58}$. Thus, activation of recurrent signaling routes within a neuronal memory circuit via DANs and the resulting sustained activity

510 in these neurons appears as a conserved mechanism to consolidate memories in insects and vertebrates.

515 


\section{Supplement figures:}
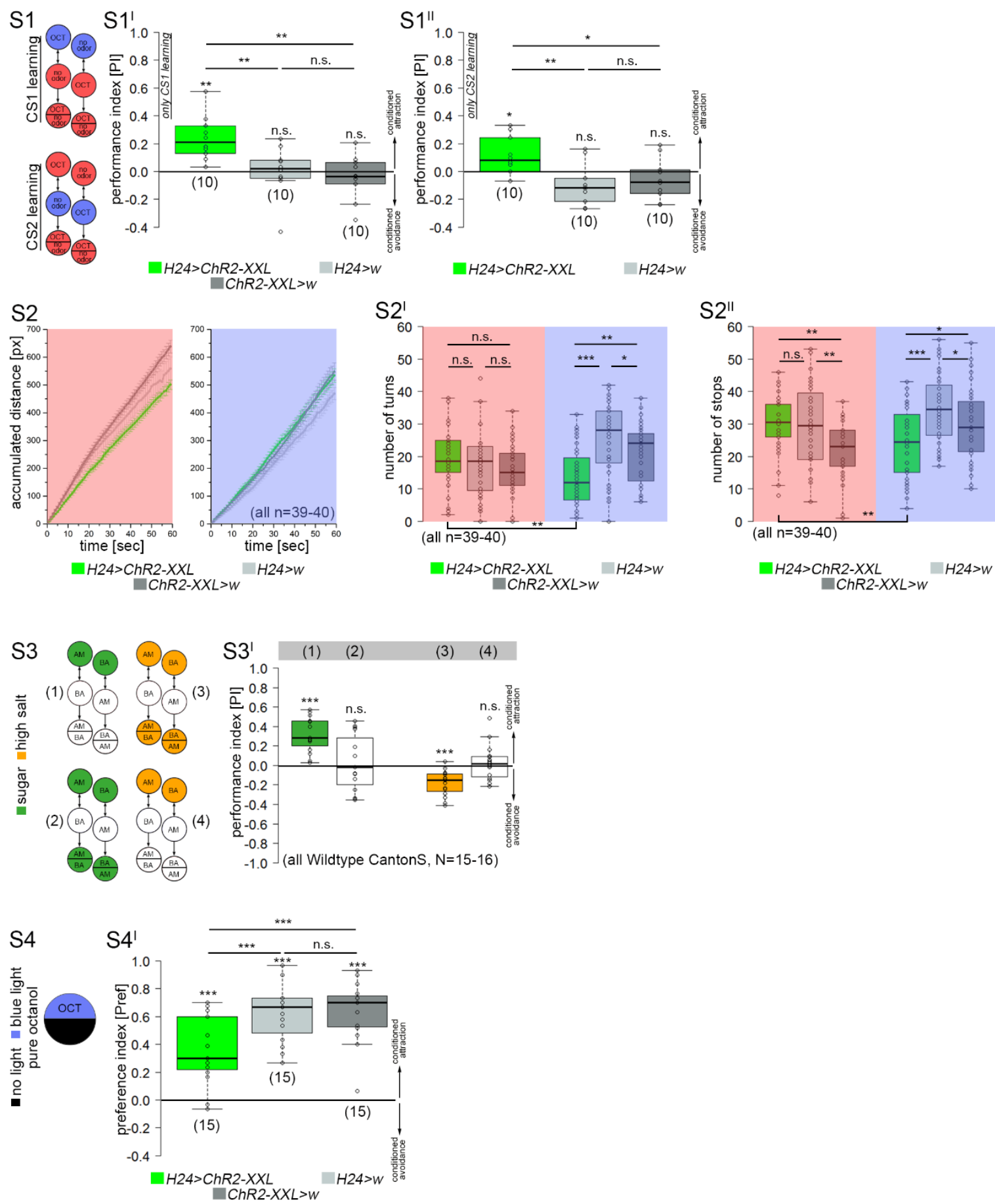

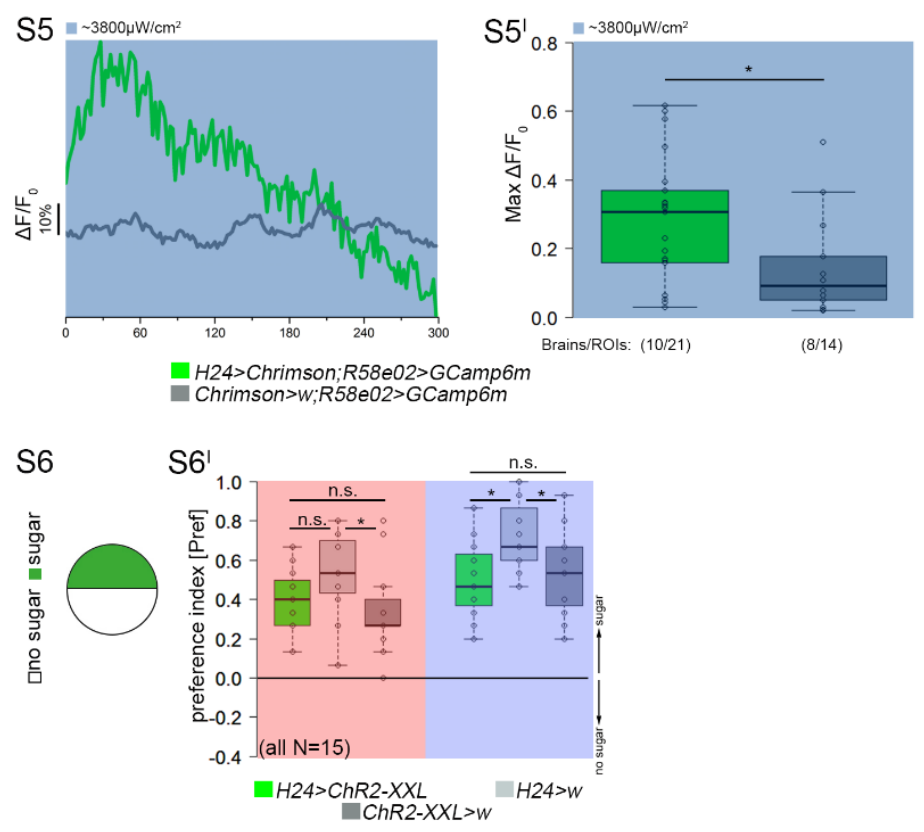

\section{Supplement figures:}

$\left(S 1-S 1^{\prime \prime}\right)$ Performance scores were independent of whether the first odor (CS1+; OCT or "no odor") or the second odor (CS2+; OCT or "no odor") was coupled to optogenetic activation of KCs during KC-substitution learning. (S2) General locomotion was unaffected by optogenetic activation of all KCs as H24>ChR2-XXL larvae crawled normal distances over time compared to genetic controls. Experimental larvae showed a reduced number of turns (S2') and stops $\left(\mathrm{S} 2^{\prime \prime}\right)$ due to blue light exposure. (S3-S3') Wildtype larvae express appetitive olfactory

550 memories not in presence of the appetitive US (e.g. sugar (green)) in the test situation, while aversive memory expression is only present when the aversive US (e.g. high salt (orange)) is absent in the test situation. (S4-S4') H24>ChR2-XXL larvae show a reduced light avoidance compared to genetic controls when they are challenged to decide between their innate OCT and darkness preference versus an optogenetically induced internal reward signaling. (S5)

555 pPAM neurons respond with a significant increase in intracellular $\mathrm{Ca}^{2+}$ levels (S5C') to an optogenetic activation of MB KCs using UAS-Chrimson (green; illumination with blue light $(475 \mathrm{~nm})$ was already sufficient for optogenetic activation via Chrimson) verifying a functional KC-to-DAN feedback connection. An obvious increase in $\mathrm{Ca}^{2+}$ levels was absent in genetic controls (grey). (S6-S6') H24>ChR2-XXL larvae showed normal innate preference for fructose

560 compared to genetic controls. AM: amylacetate; BA: benzaldehyde; ChR2-XXL: channelrhodopsin-XXL; CS: conditioned stimulus; OCT:octanol. Significance levels: $p>0.05$ n.s., $p<0.05^{*}, p<0.01^{* *}, p<0.001^{* * *}$. 


\section{References:}

1. Heisenberg, M. Mushroom body memoir: from maps to models. Nat. Rev.

Neurosci. 4, 266-275 (2003).

575 2. Gerber, B., Tanimoto, H. \& Heisenberg, M. An engram found? Evaluating the evidence from fruit flies. Curr. Opin. Neurobiol. 14, 737-744 (2004).

3. Pauls, D., Selcho, M., Gendre, N., Stocker, R. F. \& Thum, A. S. Drosophila larvae establish appetitive olfactory memories via mushroom body neurons of embryonic origin. J. Neurosci. Off. J. Soc. Neurosci. 30, 10655-10666 (2010).

580 4. Cognigni, P., Felsenberg, J. \& Waddell, S. Do the right thing: neural network mechanisms of memory formation, expression and update in Drosophila. Curr. Opin. Neurobiol. 49, 51-58 (2018).

5. Widmann, A., Eichler, K., Selcho, M., Thum, A. S. \& Pauls, D. Odor-taste learning in Drosophila larvae. J. Insect Physiol. 106, 47-54 (2018).

585 6. Aso, Y. et al. The neuronal architecture of the mushroom body provides a logic for associative learning. eLife 3, e04577 (2014).

7. Busto, G. U., Cervantes-Sandoval, I. \& Davis, R. L. Olfactory learning in Drosophila. Physiol. Bethesda Md 25, 338-346 (2010).

8. McGuire, S. E., Le, P. T. \& Davis, R. L. The role of Drosophila mushroom body signaling in olfactory memory. Science 293, 1330-1333 (2001).

9. Schleyer, M. et al. A behavior-based circuit model of how outcome expectations organize learned behavior in larval Drosophila. Learn. Mem. Cold Spring Harb. N 18, 639-653 (2011).

10. McGuire, S. E., Deshazer, M. \& Davis, R. L. Thirty years of olfactory learning and memory research in Drosophila melanogaster. Prog. Neurobiol. 76, 328-347 (2005). 
11. Eichler, K. et al. The complete connectome of a learning and memory centre in an insect brain. Nature 548, 175-182 (2017).

12. Saumweber, T. et al. Functional architecture of reward learning in mushroom body extrinsic neurons of larval Drosophila. Nat. Commun. 9, 1104 (2018).

13. Rohwedder, A. et al. Four Individually Identified Paired Dopamine Neurons Signal Reward in Larval Drosophila. Curr. Biol. CB 26, 661-669 (2016).

14. Selcho, M., Pauls, D., Han, K.-A., Stocker, R. F. \& Thum, A. S. The role of dopamine in Drosophila larval classical olfactory conditioning. PloS One 4, e5897 (2009).

15. Schroll, C. et al. Light-induced activation of distinct modulatory neurons triggers appetitive or aversive learning in Drosophila larvae. Curr. Biol. CB 16, 1741-1747 (2006).

16. Burke, C. J. et al. Layered reward signalling through octopamine and dopamine in Drosophila. Nature 492, 433-437 (2012).

17. Selcho, M., Pauls, D., Huser, A., Stocker, R. F. \& Thum, A. S. Characterization of the octopaminergic and tyraminergic neurons in the central brain of Drosophila larvae. J. Comp. Neurol. 522, 3485-3500 (2014).

18. Selcho, M., Pauls, D., El Jundi, B., Stocker, R. F. \& Thum, A. S. The role of octopamine and tyramine in Drosophila larval locomotion. J. Comp. Neurol. 520, 3764-3785 (2012).

19. Huetteroth, W. et al. Sweet taste and nutrient value subdivide rewarding dopaminergic neurons in Drosophila. Curr. Biol. CB 25, 751-758 (2015).

20. Ichinose, T. et al. Reward signal in a recurrent circuit drives appetitive long-term memory formation. eLife 4, e10719 (2015). 
21. Pavlowsky, A., Schor, J., Plaçais, P.-Y. \& Preat, T. A GABAergic Feedback Shapes Dopaminergic Input on the Drosophila Mushroom Body to Promote Appetitive Long-Term Memory. Curr. Biol. CB 28, 1783-1793.e4 (2018).

22. Saumweber, T., Husse, J. \& Gerber, B. Innate attractiveness and associative learnability of odors can be dissociated in larval Drosophila. Chem. Senses 36, 223-235 (2011).

23. Brand, A. H. \& Perrimon, N. Targeted gene expression as a means of altering cell fates and generating dominant phenotypes. Dev. Camb. Engl. 118, 401-415 (1993).

630 24. Dawydow, A. et al. Channelrhodopsin-2-XXL, a powerful optogenetic tool for lowlight applications. Proc. Natl. Acad. Sci. U. S. A. 111, 13972-13977 (2014).

25. Manoli, D. S. et al. Male-specific fruitless specifies the neural substrates of Drosophila courtship behaviour. Nature 436, 395-400 (2005).

26. Adachi, Y. et al. Conserved cis-regulatory modules mediate complex neural expression patterns of the eyeless gene in the Drosophila brain. Mech. Dev. 120, 1113-1126 (2003).

27. Risse, B. et al. FIM, a novel FTIR-based imaging method for high throughput locomotion analysis. PloS One 8, e53963 (2013).

28. von Essen, A. M. H. J., Pauls, D., Thum, A. S. \& Sprecher, S. G. Capacity of visual classical conditioning in Drosophila larvae. Behav. Neurosci. 125, 921-929 (2011).

29. Keene, A. C. \& Sprecher, S. G. Seeing the light: photobehavior in fruit fly larvae. Trends Neurosci. 35, 104-110 (2012).

30. Pauls, D. et al. Potency of transgenic effectors for neurogenetic manipulation in 645 Drosophila larvae. Genetics 199, 25-37 (2015). 
31. Warrick, J. M., Vakil, M. F. \& Tompkins, L. Spectral sensitivity of wild-type and mutant Drosophila melanogaster larvae. J. Neurogenet. 13, 145-156 (1999).

32. Humberg, T.-H. \& Sprecher, S. G. Age- and Wavelength-Dependency of Drosophila Larval Phototaxis and Behavioral Responses to Natural Lighting Conditions. Front. Behav. Neurosci. 11, 66 (2017).

33. Klapoetke, N. C. et al. Independent optical excitation of distinct neural populations. Nat. Methods 11, 338-346 (2014).

34. Schleyer, M. et al. The impact of odor-reward memory on chemotaxis in larval Drosophila. Learn. Mem. Cold Spring Harb. N 22, 267-277 (2015).

655 35. Hendel, T. et al. The carrot, not the stick: appetitive rather than aversive gustatory stimuli support associative olfactory learning in individually assayed Drosophila larvae. J. Comp. Physiol. A Neuroethol. Sens. Neural. Behav. Physiol. 191, 265279 (2005).

36. Schleyer, M., Miura, D., Tanimura, T. \& Gerber, B. Learning the specific quality of taste reinforcement in larval Drosophila. eLife 4, (2015).

37. Liu, Q., Liu, S., Kodama, L., Driscoll, M. R. \& Wu, M. N. Two dopaminergic neurons signal to the dorsal fan-shaped body to promote wakefulness in Drosophila. Curr. Biol. CB 22, 2114-2123 (2012).

38. Lai, S.-L. \& Lee, T. Genetic mosaic with dual binary transcriptional systems in Drosophila. Nat. Neurosci. 9, 703-709 (2006).

39. Feinberg, E. H. et al. GFP Reconstitution Across Synaptic Partners (GRASP) defines cell contacts and synapses in living nervous systems. Neuron 57, 353363 (2008).

40. Kim, Y.-C., Lee, H.-G. \& Han, K.-A. D1 dopamine receptor dDA1 is required in the 670 mushroom body neurons for aversive and appetitive learning in Drosophila. J. Neurosci. Off. J. Soc. Neurosci. 27, 7640-7647 (2007). 
41. Cervantes-Sandoval, I., Phan, A., Chakraborty, M. \& Davis, R. L. Reciprocal synapses between mushroom body and dopamine neurons form a positive feedback loop required for learning. eLife 6, (2017).

675 42. Tanaka, N. K., Tanimoto, H. \& Ito, K. Neuronal assemblies of the Drosophila mushroom body. J. Comp. Neurol. 508, 711-755 (2008).

43. Honjo, K. \& Furukubo-Tokunaga, K. Induction of cAMP response element-binding protein-dependent medium-term memory by appetitive gustatory reinforcement in Drosophila larvae. J. Neurosci. Off. J. Soc. Neurosci. 25, 7905-7913 (2005).

44. Widmann, A. et al. Genetic Dissection of Aversive Associative Olfactory Learning and Memory in Drosophila Larvae. PLoS Genet. 12, e1006378 (2016).

45. Cohn, R., Morantte, I. \& Ruta, V. Coordinated and Compartmentalized Neuromodulation Shapes Sensory Processing in Drosophila. Cell 163, 17421755 (2015).

685 46. Takemura, S.-Y. et al. A connectome of a learning and memory center in the adult Drosophila brain. eLife 6, (2017).

47. Zhao, X., Lenek, D., Dag, U., Dickson, B. J. \& Keleman, K. Persistent activity in a recurrent circuit underlies courtship memory in Drosophila. eLife 7, (2018).

48. Keene, A. C., Krashes, M. J., Leung, B., Bernard, J. A. \& Waddell, S. Drosophila 690 dorsal paired medial neurons provide a general mechanism for memory consolidation. Curr. Biol. CB 16, 1524-1530 (2006).

49. Musso, P.-Y., Tchenio, P. \& Preat, T. Delayed dopamine signaling of energy level builds appetitive long-term memory in Drosophila. Cell Rep. 10, 1023-1031 (2015).

695 50. Hawkins, R. D., Kandel, E. R. \& Bailey, C. H. Molecular mechanisms of memory storage in Aplysia. Biol. Bull. 210, 174-191 (2006). 
51. Blum, A. L., Li, W., Cressy, M. \& Dubnau, J. Short- and long-term memory in Drosophila require cAMP signaling in distinct neuron types. Curr. Biol. CB 19, $1341-1350$ (2009).

52. Plaçais, P.-Y. et al. Slow oscillations in two pairs of dopaminergic neurons gate long-term memory formation in Drosophila. Nat. Neurosci. 15, 592-599 (2012).

53. Plaçais, P.-Y. \& Preat, T. To favor survival under food shortage, the brain disables costly memory. Science 339, 440-442 (2013).

54. Rohwedder, A., Selcho, M., Chassot, B. \& Thum, A. S. Neuropeptide F neurons modulate sugar reward during associative olfactory learning of Drosophila larvae. J. Comp. Neurol. 523, 2637-2664 (2015).

55. Krashes, M. J. et al. A neural circuit mechanism integrating motivational state with memory expression in Drosophila. Cell 139, 416-427 (2009).

56. Waddell, S. Neural Plasticity: Dopamine Tunes the Mushroom Body Output Network. Curr. Biol. 26, R109-R112 (2016).

57. Chowdhury, R., Guitart-Masip, M., Bunzeck, N., Dolan, R. J. \& Düzel, E.

Dopamine modulates episodic memory persistence in old age. J. Neurosci. Off. J. Soc. Neurosci. 32, 14193-14204 (2012).

58. Bethus, I., Tse, D. \& Morris, R. G. M. Dopamine and memory: modulation of the persistence of memory for novel hippocampal NMDA receptor-dependent paired associates. J. Neurosci. Off. J. Soc. Neurosci. 30, 1610-1618 (2010).

59. Apostolopoulou, A. A., Widmann, A., Rohwedder, A., Pfitzenmaier, J. E. \& Thum, A. S. Appetitive associative olfactory learning in Drosophila larvae. J. Vis. Exp. JoVE (2013). doi:10.3791/4334

720 60. Selcho, M. \& Wegener, C. Immunofluorescence and Genetic Fluorescent Labeling Techniques in the Drosophila Nervous System. Immunocytochem. Relat. Tech. 101, 39-62 (2015). 
61. Feng, Y., Ueda, A. \& Wu, C.-F. A modified minimal hemolymph-like solution, HL3.1, for physiological recordings at the neuromuscular junctions of normal and mutant Drosophila larvae. J. Neurogenet. 18, 377-402 (2004).

62. Schindelin, J. et al. Fiji: an open-source platform for biological-image analysis.

Nat. Methods 9, 676-682 (2012).

\section{Material and Methods:}

\subsection{Fly strains:}

Flies were cultured according to standard methods. In short, vials were kept under constant conditions with $25^{\circ} \mathrm{C}$ and $60 \%$ humidity in a $12: 12$ light:dark cycle. Driver lines used in this study were H24-Gal4 (chromosome II), Ok107-Gal4 (IV), R58E02-Gal4 (III), and R58E02-lexA (II). UAS lines included in this study were 10xUAS-IVS735 myr::GFP (II), UAS-mCD8::GFP (II), UAS-ChR2-XXL (II), 20xUAS-CsChrimson (II), UAS-DopR1-RNAi (III), lexAop-reaper (II), and 13xLexAop-IVS-GCamp6m/20xUASCsChrimson (II,III). Genetic controls were obtained by crossing Gal4-driver/Lex-driver and UAS-effector/LexAop-effector lines to $w^{1118}$.

\section{$740 \quad 6.2$ Behavioral experiments:}

\subsubsection{Associative conditioning}

Appetitive olfactory learning was tested using standardized, previously described assays 5,59 . Learning experiments were performed on plates filled by a thin layer of pure $1.5 \%$ agarose solution (Roth, 9012-36-6). We mainly used a one-odor reciprocal training design ${ }^{22}$, where larvae were exposed to $10 \mu$ l of 1-octanol (OCT, Sigma, 11187-5). We also performed one experiment using the two-odor reciprocal training design, where $10 \mu \mathrm{l}$ of amylacetate (AM, Merck, 628-63-7) was exposed to the larvae opposing OCT. Odorants were loaded into custom-made Teflon containers $(4.5 \mathrm{~mm}$ diameter) with perforated lids.

For KC-substitution learning experiments, a first group of 30 larvae was trained to associate OCT to blue light exposure (OCT+). After $5 \mathrm{~min}$ the larvae were transferred to a second Petri dish containing no odor under red light (NO). Simultaneously, a second group of larvae was trained reciprocally with blue light exposure coupled to no 
odor (NO+/OCT). After three training cycles, larvae were immediately transferred to the test plate, where OCT was presented on one side of the dish. To test the persistence of the established memory, larvae were tested $10 \mathrm{~min}, 15 \mathrm{~min}, 30 \mathrm{~min}$, or $45 \mathrm{~min}$ after the training. After $3 \mathrm{~min}$, larvae were counted in the OCT side (\#OCT), the no odor side (\#NO), and in the neutral zone (for further details, a video is provided in $\left.{ }^{59}\right)$. The procedure was similar for the two-odor reciprocal training design (OCT+/AM and OCT/AM+). The preference index was calculated by subtracting the number of larvae on the OCT side from the number of larvae on the no odor side (or AM side), divided by the total number of animals (including the larvae counted in the neutral zone).

765

(a) PREFOCT+/NO = (\#OCT - \#NO) / \#TOTAL

(b) PREFOCT/NO+ = (\#OCT - \#NO) / \#TOTAL

Subsequently, we calculated the performance index (PI). Positive Pls indicate appetitive learning, while negative Pls indicate aversive learning.

(c) $\mathrm{PI}=(\mathrm{PREFOCT}+/ \mathrm{NO}-\mathrm{PREFOCT/NO}+) / 2$

For optogenetic manipulation in all behavioral experiments (using UAS-ChR2-XXL) we used $475 \mathrm{~nm}$ light-emitting diodes (LED) with a light intensity of $\sim 1300 \mu \mathrm{W} / \mathrm{cm}^{2}$, or red light with $620 \mathrm{~nm}$ and the intensity of $50 \mu \mathrm{W} / \mathrm{cm}^{2}$ for UAS-Chrimson. To induce optogenetic activation, the light-emitting diodes were placed $\sim 45 \mathrm{~cm}$ above the Petri dish, while all other steps of the experimental procedure were done under red light (or complete darkness for UAS-Chrimson).

\subsubsection{Olfactory preference tests}

To test larvae for their innate odor response, an odor container was placed on one side of the Petri dish containing $1.5 \%$ agarose to induce a choice test. For optogenetic manipulation the Petri dish was placed in blue light, for control experiments the choice test was done under red light. The preference index was calculated by subtracting the number of larvae on the odor side (\#Odor) from the number of larvae on the no odor side (\#NO), divided by the total number of animals (including the larvae counted in the neutral zone). In each test, we used a group of 30 larvae. 
PREFOdor/NO = (\#Odor $-\# N O) / \# T O T A L$

Negative PREF values indicate an avoidance of the odor, whereas positive PREF values represent an attractive response.

\section{$795 \quad$ 6.2.3 Gustatory preference tests}

To test larvae for their innate gustatory response during optogenetic activation, one half of the Petri dish was filled with $1.5 \%$ pure agarose, while the other half was filled with 1.5\% agarose containing $2 \mathrm{M}$ fructose (Roth, 57-48-7). For optogenetic manipulation the Petri dish was placed in blue light, for control experiments the choice

800 test was done under red light. The preference index was calculated by subtracting the number of larvae on the sugar side (\#Sugar) from the number of larvae on the no sugar side (\#NS), divided by the total number of animals (including the larvae counted in the neutral zone). In each test, we used a group of 30 larvae.

PREFsugar/NS = (\#Sugar - \#NO) $/$ \#TOTAL

Negative PREF values indicate sugar avoidance, whereas positive PREF values represent an attractive response.

\section{$810 \quad$ 6.2.4 Light preference tests}

To test larvae for their response to blue or red light, respectively, (and by that to optogenetic manipulation), the Petri dish containing $1.5 \%$ agarose was covered by a lid, divided in two transparent and two shaded quarters, respectively. The preference index was calculated by subtracting the number of larvae on the dark side (\#DS) from

815 the number of larvae on the illuminated side (\#Light), divided by the total number of animals (including the larvae counted in the neutral zone). In each test, we used a group of 30 larvae.

PREFDS = (\#DS - \#Light) $/$ \#TOTAL

Positive PREF values indicate light avoidance, whereas negative PREF values represent approach towards the illuminated side. 


\subsection{Locomotion assay:}

825 For the locomotion assay we used the FIM (ㅌTIR-based Imaging Method) tracking system as described in ${ }^{27}$. Recordings were made by a monochrome industrial camera (DMK27BUP031) with a Pentax C2514-M objective in combination with a Schneider infrared pass filter, and the IC capture software (www.theimagingsource.com). To analyze larval locomotion, a group of 10 larvae was recorded on $1.5 \%$ agarose for two

830 minutes. During the first minute, larvae were exposed to red light, while they were exposed to blue light for the second minute for optogenetic activation of KCs. We analyzed the following parameters using the FIM track software: accumulated distance, velocity, number of stops, and number of bendings.

\subsection{Immunofluorescence:}

Immunofluorescence studies were performed as described in ${ }^{60}$. In short, 5-6 day old larvae were dissected in phosphate buffer saline (PBS) or HL3.1 (pH 7.2) ${ }^{61}$, fixated in $4 \%$ paraformaldehyde in PBS for 40min, washed four times in PBS with $0.3 \%$ Triton-X 100 (PBT), and afterwards blocked in 5\% normal goat serum in PBT. Specimens were

840 incubated in primary antibody solution containing polyclonal rabbit anti-GFP antibody (A6455, Molecular Probes, dilution 1:1000) in blocking solution for one night at $4^{\circ} \mathrm{C}$. Then brains were washed six times in PBT and incubated for one night at $4{ }^{\circ} \mathrm{C}$ in secondary antibody solution containing goat anti-rabbit Alexa 488 (Molecular Probes, dilution 1:250). Finally, specimens were rinsed six times in PBT and mounted in $80 \%$

845 glycerol in PBS. Until scanning with a Leica SP8 confocal light scanning microscope, brains were stored in darkness at $4^{\circ} \mathrm{C}$. Image processing was performed with $\mathrm{Fiji} 62$ and Adobe Photoshop CS6 (Adobe Systems, San Jose, CA).

\subsection{Functional imaging:}

850 To monitor intracellular $\mathrm{Ca}^{2+}$ levels in dopaminergic neurons of the pPAM cluster in response to the optogenetic activation of MB KCs, LexAop-GCaMP6m was expressed via R58E02-LexA and UAS-ChR2-XXL via H24-Gal4. 1-2 larval brains were dissected and subsequently put in a Petri dish containing 405 $\mu$ l hemolymph-like HL3.1 saline solution. Before the experiment, brains maintained for around $30 \mathrm{~min}$ for settling. 855 Specimens were imaged with an AXIO Examiner D1 upright microscope (Carl Zeiss AG, Germany) with a Cairn Twin-Cam LS image splitter, two pco.edge 4.2 sCMOS 
cameras, and a SPECTRA-4 light engine. Images were taken with a Zeiss W PlanApochromat $x 20 / 1.0$ or $x 40 / 1.0$ water immersion objective.

During monitoring, brains were first excited with 475nm light and an exposure time of was increased to around $3800 \mu \mathrm{W} / \mathrm{cm}^{2}$ for optogenetic activation of MB KCs. We continued the monitoring of $\mathrm{Ca}^{2+}$ levels in pPAM neurons for $5 \mathrm{~min}$.

Similarly, MB KCs were optogenetically activated via UAS-Chrimson. Here, we recorded $\mathrm{Ca}^{2+}$ levels in R58E02> GCaMP6m brain with $475 \mathrm{~nm}$ light and an intensity of $3800 \mu \mathrm{W} / \mathrm{cm}^{2}$, but without pre-exposure of a lower light intensity.

\subsection{Statistical methods:}

Data was analyzed for normal distribution using the Shapiro-Wilk Normality test. To test against chance level, a t-test was used for normally distributed data, a Wilcoxon

870 Signed Rank test for not normally distributed data. For the comparison between genotypes, a pairwise t-test was used for normally distributed data, a pairwise Wilcoxon Rank Sum test was used for not normally distributed data. Pairwise tests included the Bonferroni-Holm correction. All statistical analyses were done with $\mathrm{R}$ Studio version 0.99.896 (www.r-project.org). Data plots were done with OriginPro

875 2016G, b9.3.226 (www.originlab.com). Data is mainly presented as box plots, with $50 \%$ of the values of a given genotype being located within the box, and whiskers represent the entire set of data. No data was excluded. Outliers are indicated as open circles. The median performance index is indicated as a thick line within the box plot. For the persistence of memory, data is also presented as a line chart, with mean values and 880 the standard error of the mean. Significance levels between genotypes shown in the figures refer to the raw $p$-values obtained in the statistical tests.

\section{Acknowledgements:}

We thank Konrad Öchsner, for technical assistance, Wolf Hütteroth, Katharina Eichler, 885 Björn Brembs, Thomas Raabe, Tim Humberg, and Martin Strube-Bloss for fruitful discussions and/or comments on the manuscript. We thank Robert Kittel, Georg Nagel, Katharina Eichler, Vivek Jayaraman, the Vienna Drosophila resource center, and the Bloomington Stock center for providing flies. The authors declare no competing interests. R.L., A.S.T. and D.P. conceived and designed the experiments. R.L., M.S., 890 M.P., J.H., D.S. and D.P. performed the experiments. R.L., M.S., C.W. and D.P. 
analyzed the results. R.L., C.W., A.S.T., and D.P. wrote the manuscript. This work was supported by the PostDoc Plus fellowship (to D.P.) and a PhD fellowship (to R.L.) from the German Excellence Initiative to the Graduate School of Life Sciences, University of Würzburg, and by the Deutsche Forschungsgemeinschaft (TH1584/1-3 to A.S.T., 895 INST 93/824-1 LAGG to C.W., and DP1979/2-1 to D.P.) and intramural funds by the University of Würzburg (to C.W.). 